since, even in the absence of $D z, K$ is incompletely dominant in some genetic brokgrounds. In the presence of $D z$, large amounts of pelargonidin are present in the flowers of $K$-genotypes, but the foliar parts only contain traces of this compound.

Flavonoids with hydroxyl (or methoxyl) groups in the $3^{\prime}, 4^{\prime}$ and $5^{\prime}$ positions, for example, myricetin and delphinidin, are absent from the recessive $k k$ genotypes, namely, $k k d z d z$ and $k k D z D z$. The latter genotype has the higher absolute content of pigment in the flowers. Both contain mainly pelargonidin, together with a small amount of peonidin (about 10 per cent) and traces of cyanidin in their petals. The foliar parts, however, contain equal amounts of pelargonidin and cyanidin. Kæmpferol and quercetin occur in all parts of the plant. In the flowers, the former predominates, and quercetin is often absent. The situation is reversed in the leaves, where again quercetin is found most abundantly.

Other related polyphenolic compounds are also present in $P$. sinensis. An unidentified flavanone glycoside, sinensin ${ }^{6}$, occurs in large quantitios in the flowers of $k k D z D z$ forms, from which it has been isolated. The aglycone, sinensetin, does not correspond in its properties with any of the commonly occurring flavanones and therefore its structure is under investigation.

The co-occurrence under gene control of anthocyanidins and flavonols having the same hydroxylation pattern was first noted by Geissman et al. ${ }^{7}$ in Antirrhinum majus and Dianthus caryophyllus, where the pairs of pigments, cyanidin and quercetin, and pelar. gonidin and kæmpferol, co-occur in different genotypes. The co-occurrence of anthocyanidins and flavonols at all three levels of hydroxylation of the $B$ ring was first observed by one of $u^{8}$ in Solanum species. $P$. sinensis thus provides a fourth example, the situation being very similar to that in the Solanum series. This work, then, supports the theory that flavonols and anthocyanidins are derived from a common precursor, have a similar biosynthetic path. way and only diverge at a very late stage in pigment synthesis.

It is also apparent that in $P$. sinensis the effects of some genes controlling pigment metabolism differ in different parts of the same plant. The only other known case of this phenomenon is that of plants of the tuberous Solanums, where tuber and flower contain different anthocyanins ${ }^{2}$. It is hoped to publish full details of this and other work elsewhere. J. B. HARBORNE H. S. A. SherratT

${ }^{2}$ De Winton, D., and Haldane, J. B. S., J. Genet., 27, 1 (1933).

2 Scott-Moncrieff, R., J. Genet., 32, 117 (1936).

${ }^{8}$ Bate-Smith, E. C., Biochem. J., 58, 122 (1954); Sci. Proc. Roy. Dublin Soc., 27, 165 (1956).

- Harborne, J. B., Chem. and Indust., 1142 (1954). Geissman, T. A. Jorgensen, E.' C., and Harborne, J. B., Chem. and Indust., 1389

s Dayton, T. O., Ph.D. thesis, London (1954).

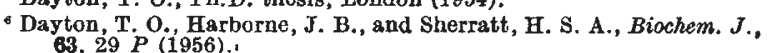

"Geissman, T. A., and Mehlquist, G. A. L., Genetics, 32, 410 (1947). Geissman. T. A., Jorgensen, E. C., and Johnson, B. L., Arch.
Biochem. and Biophys., 49, 368 (1954).

${ }^{8}$ Harborne, J. B., John Innes Ann. Rep., 20 (1956).

- Dodds, K. S., and Long, D. H., J. Genet., 53, 136 (1955).

\section{Anthocyanins in the Flowers of Primula sinensis}

STudies were made previously of the biochemical genetics of the flower pigments of Primula sinensis ${ }^{1}$. A erystalline anthoeyanin from red flowers, 'primulin', was stated to be malvidin 3-galactoside on the basis of its distribution number between pentanol and water ${ }^{2}$, and more recently the pelargonidin derivative present in orange flowers (genotype $k k D z D z$ ) was stated to be a 3 -galactoside ${ }^{3}$.

As part of a re-investigation of the polyphenolic componentrs of $P$. sinensis ${ }^{4}$, the anthocyanins of $K K d z d z$ and $k k D z D z$ flowers were examined by paper chromatography. Extracts of flowers in methanolic hydrochloric acid were banded on Whatman No. 3 paper and developed with $n$-butanol/2N hydrochloric acid 1/1 (upper layer). The separated anthocyanin bands were cut out and eluted with 5 per cent acetic acid in methanol and purified by re-running in $n$-butanol/acetic acid/water, 4/1/5 (upper layer), and then 15 per cent aqueous acetic acid on washed paper. The purified pigments were hydrolysed and the aglycone and sugar components identified ${ }^{3}$.

The anthocyanins in $K K$ flowers were found to be malvidin 3-glucoside with an appreciable amount of petunidin 3-glucoside. Orange $(k k D z D z)$ flowers contained pelargonidin 3-glucoside, smaller amounts of peonidin 3 -glucoside (about 10 per cent) and much smaller amounts of a cyanidin 3-hexoside (about 1 per cent); they also contrined two other pelargonidin glucosides which did not correspond in their chromatographic behaviour in five solvent systems with any known pelargonidin derivative.

No galactose was detected in the hydrolysates of any of the anthocyanins. It was, however, readily confirmed by these methods that cyanidin 3-galactoside was present in the leaves of the copper beech, Fagus sylvatica, ${ }^{5,6}$. A few milligrams of the crystalline anthocyanin, 'primulin', isolated from red flowers of $P$. sinensis, and also a crystalline anthocyanin isolated from magenta flowers ${ }^{1}$, were kindly made available by Miss V. C. Sturgess. These were both found to yield only glucose on hydrolysis and to consist of malvidin 3-glucoside containing about 15 per cent of petunidin 3-glucoside. This material would therefore be expected to give a different distribution number from that of malvidin 3-glucoside, which might explain the earlier incorrect identification of 'primulin' as the 3-galactoside.

Anthocyanins which have been isolated so far from flowers of other members of the Primulaceas are all glucosides, namely, malvidin 3-glucoside from $P$. polyanthus ${ }^{7}$ malvidin 3:5-diglucoside from $P$. viscosa and $P$. integrifola ${ }^{8}$, delphinidin 3 : 5-diglucoside from $P$. obconica ${ }^{2}$ and hirsutidin $3: 5$-diglucoside from $P$. hirsuta ${ }^{8}$. It is no longer necessary to retain the name 'primulin's for the supposed malvidin 3.galactoside from $P$. sinensis.

\section{H. S. A. SherratT}

${ }^{1}$ Scott-Moncrieff, R., J. Genet., 32, 117 (1936).

2 Bell, J. C., and Robinson, R., J. Chem. Soc., 813 (1934).

"Harborne, J. B., and Sherratt, H. S. A., Biochem. J., 65, 23 P (1957).

- Dayton, T. O., Harborne, J. B., and Sherratt, H. S. A., Biochem. J., 63, 29 P (i956).

'Robinson, G. M., and Robinson, R., Biochem. J., 26, 1647 (1932).

- Smith, H., and Robinson, R., Nature, 175, 634 (1955).

'Scott-Moncrieff, R., Biochem. J., 24, 767 (1930).

- Karrer, P., and Widmer, R., Helv. Chim. Acta, 10, 14, 758 (1927).

- Harborne, J. B. (unpublished).

\section{Anthocyanidins of the Primulaceae}

IN connexion with detailed chemical and genetical investigations of the flavonoid pigments of Primula species, a brief survey of the anthocyanidins present 